\title{
Professor Jun Li Treating Vascular Dementia from Mutual Conclusion of Phlegm and Blood Stasis
}

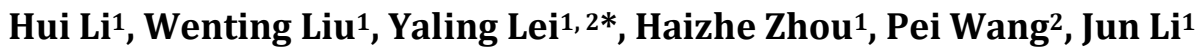 \\ 1Shaanxi University of Chinese Medicine, Xianyang 712046, Shaanxi Province, China \\ ${ }^{2}$ Shaanxi Provincial Hospital of Chinese Medicine, Xi'an 710003, Shaanxi Province, China \\ *Corresponding author: Yaling Lei, ia678@qq.com
}

Copyright: () 2022 Author(s). This is an open-access article distributed under the terms of the Creative Commons Attribution License (CC BY 4.0), permitting distribution and reproduction in any medium, provided the original work is cited.

\begin{abstract}
Professor Jun Li believes that the main etiology and pathogenesis of vascular dementia is the combination of phlegm and blood stasis. The symptoms include feeling faint and declined senses in organs of the head, caused by mystery reason. To cure phlegm and blood stasis simultaneously, the therapy promotes the notion of "resolving phlegm and stimulating the circulation of blood, liberate brain orifices," and treatments with the addition and removal of "Naotaitong Decoction," which has an amazing clinical curative effect. This paper examines Professor Jun Li's theoretical foundations and ideas from the syndrome differentiation and treatment of phlegm and blood stasis, systematically examines the curative effect and mechanism of Naotaitong Decoction in the treatment of vascular dementia, and examines the prescription and medication of typical cases for readers, in the hopes of providing guidance to clinical diagnosis and treatment of this disease.
\end{abstract}

Keywords: Professor Jun Li; Phlegm and blood stasis; Vascular dementia; Diagnosis and treatment experience

Online publication: January 19, 2022

\section{Introduction}

Vascular Dementia (VD) is a clinical syndrome caused by various cerebrovascular diseases and risk factors, with memory loss, cognitive and executive function decline, and even abnormal behavior as the main symptoms ${ }^{[1]}$. VD is common in elderly patients. The incidence rate of these diseases increases with the aging society and cerebrovascular disease incidence. The incidence rate of elderly patients with ischemic stroke is nearly $30 \%{ }^{[2]}$. At present, conventional western medicine treatment mainly focuses on prevention, with long treatment courses, unsatisfactory effects and adverse reactions ${ }^{[3]}$. More studies have shown that ${ }^{[4-9]}$, traditional Chinese medicine has advantages in improving cognitive symptoms, improving activities of daily living and treatment efficiency in the treatment of VD, and no risk of adverse reactions has been found.

Professor Jun $\mathrm{Li}$ is a PhD supervisor and a disciple of Zhang Xuewen, a master of Traditional Chinese medicine, who has been asked to pass on their academic expertise to the fifth and sixth batches of senior Chinese medicine professionals in China. Professor Li developed the theoretical system of brain and viscera in traditional Chinese medicine ${ }^{[10]}$, proposed the theory of "brain phlegm and blood stasis syndrome" [11], summarized the pathological characteristics of "brain is the orifice of the clearing soul - the orifice is easy to close," and argued that the value of brain orifices lies in clearing the soul and channeling benefits. Various disorders will develop if the orifices in the brain are closed ${ }^{[10]}$. Professor Li believes that the pathogenesis of VD is precisely caused by phlegm and blood stasis, which are intertwined with each other, 
or wet hot water is mixed with evil to close the orifices and clear the orifices, so there are main symptoms and manifestations such as amnesia and dementia; Syndrome differentiation and treatment should emphasize the treatment idea of thinking of blood stasis when seeing phlegm and thinking of phlegm when seeing blood stasis ${ }^{[12]}$. The method of simultaneous treatment of phlegm and blood stasis based on "transforming phlegm and Qi, promoting blood stasis and water, and dredging brain orifices" is adopted, and his self-made prescription "Naotaitong Decoction" is added and subtracted to achieve the effects of resolving phlegm and Qi, promoting blood circulation and water, opening orifices and refreshing brain. After clinical practice, the symptoms of VD patients have improved significantly. The author has the honor to listen to Professor Li Jun's clinical guidance and teaching. Now we will share our experience with you.

\section{Etiology and pathogenesis}

VD belongs to the category of "amnesia, dementia and civil madness" in traditional Chinese medicine. Its etiology and pathogenesis are mostly considered as "brain pulse stasis, kidney deficiency and marrow deficiency" ${ }^{[3]}$. Professor Jun Li advocates that the etiology and pathogenesis of VD are mainly phlegm and blood stasis blocking the orifices and mysterious mechanism losing nourishment. Its disease is located in the brain and is closely related to kidney deficiency and essence deficiency. Analysis of Professor Li's clinical experience for many years:

(1) Dementia occurs when the blood and Qi of the elderly run slowly, the blood vessels are stagnant, and the blood stasis is blocked in clearing the orifices.

(2) Modern people's diet structure is mostly the product of greasy and surfeit flavor, the function of spleen and stomach transportation is blocked, the evil of dampness and turbidity gradually accumulates phlegm and condenses phlegm into blood stasis, phlegm and blood stasis block collaterals, brain collaterals are blocked, and the spirit is unclear, which leads to dementia.

(3) Most patients with clinical syndrome are accompanied by stroke and vertigo. If they have been ill for a long time, their normal Qi is consumed, their Qi deficiency is unable to transport blood, their blood circulation is slow, and gradually becomes blood stasis. Their body fluid and blood have the same origin, and then their body fluid is deficient. Their liquid refining is phlegm, which leads to phlegm and blood stasis, which interact with each other, and their brain orifices lose nourishment, resulting in dementia.

(4) VD is often seen in elderly and chronically ill patients. Blood stasis and phlegm turbidity are often combined as diseases, hurting the viscera, damaging the healthy Qi for a long time, resulting in the deficiency of all five internal organs, especially the deficiency of kidney and essence, resulting in the loss of nourishment of brain marrow, reduction of marrow and elimination of brain, causing the lack of magic mechanism, brain deafness and resulting in dementia.

(5) The brain is the house of the primordial spirit. Clearing and unblocking is important for brain orifices, and blood stasis is blocked in the brain collaterals. Blood stasis can form edema. Phlegm and edema are combined, and blood stasis is retained in the brain. The brain water stasis is formed, the brain orifices are out of nourishment, and the loss of the primordial spirit is dementia.

\section{Theory and ideas of syndrome differentiation and treatment}

\subsection{Treat according to phlegm, sweep phlegm, arouse the brain, move Qi and open orifices}

Dementia can be caused by phlegm turbidity. "When phlegm is the most abundant, dementia Qi is the profound," according to the "Secret Records of the Stone Room." [13]. "If the heart is consumed with phlegm," Jingyue Zhang stated, "people would forget more in the event of trouble" [14] "Blood stasis on the top causes individuals to forget," according to "Standards of Diagnosis and Treatment" [15]. PPHlegm confuses the heart, leading in "crazy speech and forgetfulness," according to the "Shi Yi De Xiao Fang 
Amnesia" ${ }^{[16]}$. Hui Han et al., ${ }^{[17]}$ investigated the efficacy of VD treatment and discovered that clinical usage of Shenqi Jiangzhuo decoction can enhance patients' cognitive performance and minimize neurotransmitter secretion regulation. Chao Yang ${ }^{[18]}$ studied the VD rat model and discovered that Ditan Decoction may significantly improve rats' learning and memory abilities. Professor Jun Li thinks that bad phlegm runs down the meridians, produces a variety of ailments, spreads throughout the body, and collects in the brain collaterals. Those who are primarily concerned with the ill of phlegm turbidity should treat phlegm with Qi. They should employ orange peel and Rhizoma Pinellinae Praeparata to eliminate moisture, increase qi, and resolve phlegm, according to "Naotaitong Decoction." To minimise phlegm and turbidity, those who lose nourishment of brain orifices owing to excessive phlegm turbidity should be treated with arisaema cum bile, concretio silicea bambusae, and Caulis Bambusae in Taenia.

\subsection{Treatment from blood stasis, brain water stasis, invigorate blood and induce diuresis}

Blood stasis can cause dementia. The ancients have long known that the "Internal Classics" said: "Blood is above, and Qi is below, which cause upset and irritated, blood is below, Qi is above, which cause chaotic and amnesia." "Treatise on Febrile Diseases: Distinguishing and Treating Pulse Syndrome of Yangming Disease" says that "those who like to forget must have blood stasis... There is blood stasis for a long time, so they like to forget." Additionally, the prescription of Taohe Chengqi Decoction should be use; "Zhizhifang · Xuerong Weiqi Hua" says: "Blood is suffering, stored in the upper, which makes people amnesia, whild blood stored in the lower makes people crazy." "Xuezhenglun" by Rongchuan Tang says: "If there is blood stasis in the heart, it also makes amnesia," "if the blood is on the top, it will be turbid and unknown." Modern doctors have also studied VD caused by blood stasis. Junsheng Wang ${ }^{[19]}$ found that Tongqiao Jianbu Decoction can improve the cognitive ability of VD model rats. Minfang Qi ${ }^{[20]}$ and others found that tanshinol may improve the cognitive function of VD model mice by regulating PI3K / Akt signaling pathway and related cytokines. Professor Li believes that the evil of blood stasis blocks the brain collaterals, closes and clears the orifices, and the brain collaterals are blocked. Blood stasis can form edema. Blood stasis and water knots cause brain water stasis. In treatment, we should promote blood circulation, remove blood stasis and promote water movement. Based on Naotaitong decoction, we should add lalang grass rhizome, herba lycopi and rhizoma alismatis to facilitate water. If the brain orifices are blocked due to severe blood stasis, we should add leech, rhizoma acori graminei, borneol and musk to promote blood circulation and dredge the orifices.

\subsection{Simultaneous treatment of phlegm and blood stasis with two pronged approaches to check erroneous ideas at the outset}

The Orthodox Tradition of Medicine says: "Qi is evil and depressed, body fluid is thick and sticky, the water in the body stays or injects... Blood is turbid", indicating that phlegm can cause blood stasis ${ }^{[21]}$; "General Treatise on the Causes and Symptoms of Diseases" says: "All kinds of phlegm are blocked by blood vessels. Thus, phlegm is also formed" ${ }^{[21]}$. It can be seen that blood stasis can also produce phlegm. Professor Li believes that phlegm and blood stasis are not only the main cause, but also the pathological product. The simultaneous treatment of phlegm and blood stasis emphasizes "thinking of blood stasis when seeing phlegm and thinking of phlegm when seeing blood stasis." In the early stage of disease development, we should not only check inconspicuous signs of phlegm and blood stasis, but also take into account the trend of phlegm and blood stasis in the long-term pathological process to achieve nipping evil in the bud. For those with severe phlegm and blood stasis, Naotaitong decoction is used to vary, and Erchen decoction is used to remove blood stasis and phlegm at the same time, so as to remove blood stasis and phlegm. In the process of diagnosis and treatment, Professor Li paid attention to the characteristics of tongue and pulse changes of patients with craniocerebral phlegm and blood stasis syndrome (especially the changes of 
sublingual context), so as to make a diagnosis of phlegm and blood stasis syndrome: White greasy, yellow greasy, or gray black greasy fur is common on the tongue. The vein under the tongue is tortuous $(++)$, and ecchymosis, petechiae or blood stasis are scattered under the tongue. The pulse can be seen as smooth, hard or thin and astringent. If the tongue body is fat and has teeth marks on the side, if the tongue is purple or dark and the coated is greasy, it is actually phlegm and blood stasis intertwined, and it must focus on expelling evil as well as must not be replenished. If lip and tongue is purple and dark, with thin white coated, fine and sink pulse which suggests Qi deficiency and blood stasis, it should be treated with invigorate Qi, promote blood circulation and remove meridian obstruction. If the tongue is dark, red and the coated is less and mottled, tongue scattered stasis silk stasis point, it is mostly stomach Yin deficiency, Qi and blood deficiency, and treatment should benefit Yin and blood circulation, reduce phlegm and dredge collaterals.

\subsection{Treat both main symptoms and accompanied symptoms, cold and heat, deficiency and excess, and medication should be used properly}

Professor Li's diagnosis and treatment of patients mainly focused on phlegm and blood stasis. At the same time, he paid attention to the identification of cold and heat deficiency and excess syndrome, and advocated treating both main and accompanied symptoms together. If there are phlegm and blood stasis, cremation heat and disturbance of clearing orifices, chlorite schist, buffalo horn, antelope's horn, arisaema cum bile, Tianzhu concretio silicea bambusae, Caulis Bambusae in Taenia etc. can be added appropriately; If phlegm and blood stasis persist for a long time, Qi deficiency, clear orifices losing nourishment, Poria cocos, Rhizoma Atractylodis Macrocephalae, Radix Codonopsis, roasted Radix Astragali seu Hedysari, etc. can be added appropriately; If Qi stagnation becomes blood stasis for a long time, body fluid is refined and gathered into phlegm, and the spirit is closed, dried orangepeel, Radix Curcumae, Rhizoma Cyperi, Radix Bupleuri, Rhizome Pinelliae Preparata, etc. can be added appropriately; With liver heat, felwort, Fructus Gardeniae, uncaria rhynchophylla, spica prunellae, rhizoma alismatis, etc. should be added appropriately.

And with solid-fu due to intestine-heat, cassia seed, raw rhubarb (lower back), mirabilite (Chongfu), whole Trichosanthes, etc. can be used. For patients with blood stasis blocking and water-infusion brain orifice not scattered, musk (or radix angelicae and borneol), lalang grass rhizome, Leonurus, radix cyathulae, etc. shall be added appropriately. Scorpion, centipede, Bombyx Batryticatus, Leech, arisaema cum bile, rhizoma sparganii, curcuma zedoary, Scutellariae Barbata, and Poria cocos were selected as appropriate for those with long-term phlegm and blood stasis and toxic stagnation of brain collaterals. For patients with chronic kidney essence deficiency and brain marrow malnutrition, deer-horn glue, Epimedium, Semen Cuscutae, Cornus officinalis, etc., should be given as appropriate, and it should be reminded that most Chinese herbal medicines filled with essence and kidney are greasy to hinder the stomach, hinder the Qi movement of spleen and stomach, and block the dredging of phlegm turbidity and blood stasis. Invisible phlegm turbidity is not dispelled, and blood stasis is difficult to resolve. Therefore, it is emphasized that at the same time of tonifying kidney and filling essence, we should pay attention to soothing liver and spleen Qi, and add liver-clearing and Qi-regulating products such as Gastrodia elata, Uncaria rhynchophylla, Radix Bupleuri, and dried orangepeel as appropriate, so as to make up for the deficiency without stagnation, so that Qi circulation leads to blood circulation, and Qi circulation leads to phlegm dissipation. In this way, phlegm and blood stasis can be treated together, the evil of phlegm and turbidity can be warmed, and blood stasis can be eliminated. In short, first treat the symptoms of phlegm turbidity and blood stasis, and then replenish the deficiencies of the previous days, so as to treat the symptoms together, so as to prevent the disadvantages of incomplete and beneficial treatment.

\section{Examples of clinical medical records}

Han, a female, 54 years old, is a farmer, and was first diagnosed on June 20, 2020 with "memory loss for 
more than half a year" as the main complaint. Half a year ago, the patient had memory loss and a sense of dullness in responding to questions. During hospitalization in the local hospital, she checked: Homocysteine $24 \mathrm{mmol} / \mathrm{L}$. Low density lipoprotein $3.21 \mathrm{mmol} / \mathrm{L}$. DSA showed that the $\mathrm{P}_{2}$ segment of the right posterior cerebral artery was occluded, and the aneurysm of the left internal carotid artery was about $2.9 \mathrm{~mm} * 6.1$ $\mathrm{mm}$.

Brain MRI: bilateral brain atrophy, bilateral cerebral hemisphere, basal ganglia, thalamus, pons multiple lacunar infarction and microbleeds. The patient was discharged from the hospital after 8 days of hospitalization. The patient had a history of hypertension for 15 years, and the blood pressure was generally controlled.

Diagnosis: the language is not fluent, the reaction is slow, accompanied by dizziness and fatigue, acquaintance is still clear, the speech logic is clear, there is no reversal of speech, can eat, sleep, and the two stools is regular. She was checked BP: $130 / 80 \mathrm{mmHg}$, with a clear mind and poor spirit. Speciality check-ups are: cranial nerve (-), muscle strength of limbs grade V, normal muscle tension, decreased memory, poor calculation power, acceptable understanding, space and reading ability, no obvious aphasia, disuse, visual space and other structural obstacles, physiological reflex, pathological reflex not drawn out, Blessed Dementia Scale score of 30 points. The complexion is green and black, the lips and tongues are purple and dark, the coated tongue is yellow, the veins under the tongue are tortuous, the veins are heavy and the strings are smooth.

(1) TCM diagnosis was: Dementia

(2) Syndrome differentiation was: Phlegm and blood stasis are combined with brain collaterals and liver heat.

(3) Treatment was: reducing the phlegm and dredging collaterals, promoting blood circulation to remove blood stasis and clearing liver heat.

(4) Prescription was: varied Naoaitong Decoction.

(5) Medical was: Dried orangepeel 15g, Rhizome Pinelliae Preparata 12g, Semen Persicae 15g, Flos Carthami 12g, acorus tatarinowii $8 \mathrm{~g}$, cortex moutan $15 \mathrm{~g}$, Rhizoma gastrodia $15 \mathrm{~g}$, uncaria rhynchophylla $15 \mathrm{~g}$, Uncaria $15 \mathrm{~g}$ (back bottom) Pueraria lobata 18g, Bulbus Fritillariae Thunbergii 12g, Thunberg Fritillary Bulb 10g, Fructus Gardeniae 12g, Rhodiola rosea 20g, fried sessile stemona root $10 \mathrm{~g}$, ground beeltle10g, Earth worm 15g, leech 5g, Radix Angelicae Sinensis 12g, charred triplet 15g each, radix angelicae $8 \mathrm{~g}$, and Rhizoma Ligustici $12 \mathrm{~g}$.

(6) There are a total of 12 doses, 1 dose per day, were taken with water and warmly taken once in the morning and evening.

On July 4, 2020, the second diagnosis was made, and the symptoms improved after taking the medicine. The patients are still forgetful, more sensitive than before, dizziness and fatigue were improved, and the tongue and pulse were the same as before. On the basis of previous prescription, add $12 \mathrm{~g}$ of Radix Curcumae and $10 \mathrm{~g}$ of Acorus tatarinowii. After taking 12 doses, her memory decline improved, her language was slightly less fluent, her slow response was reduced, her blood pressure was well controlled, and her fatigue and dizziness significantly improved.

On July 20, 2020, she was diagnosed for the third time and was still amnesia, slightly slow in response, has a slightly dry cough recently, and her tongue and pulse were the same as before. Then add 10g Cortex Mori on the basis of the previous prescription, and take 12 doses. The memory decline improved.

On August 5, 2020, she was diagnosed for the fourth time and complained of improved dry cough, improved amnesia, red tongue, yellow coated tongue, deep pulse and slip string. On the basis of the previous prescription, Cortex Mori, Semen Persicae and Flos Carthami reduced to 8g, radix angelicae increased to $10 \mathrm{~g}$, followed by 12 doses, her memory decreased and improved, and her language was a little less fluent.

On August 25, 2020, she was diagnosed for the fifth times. The amnesia improved after taking the drug, 
the reaction became more sensitive, and the tongue and pulse were better than before. It was suggested that the prior prescription be supplemented with $10 \mathrm{~g}$ of Polygonatum Kingianum and $10 \mathrm{~g}$ of wine Cornus, followed by 12 doses. Memory loss was minimized, delayed responses were reduced, language was fluent, blood pressure was well managed, and there were no signs of exhaustion or dizziness.

Following a one-month follow-up, the patient's memory had improved, his calculation power had improved, his language had improved, his Blessed Dementia Scale score had improved to 30, his tongue and pulse had improved, his blood pressure had improved, and his quality of life and clinical symptoms had improved. She can follow up if she feels discomforted.

\subsection{Note}

For the past 15 years, the patient has been suffering from hypertension. She always craves sugary, oily, and thick foods. She's a tad cranky. The spleen loses its health and motility if she eats sugary, oily, and thick foods, and phlegm is produced endogenously. The majority of her irritation is caused by liver Qi stagnation, which causes Qi depression to convert into fire. In the heat of evil, blood is thick, and phlegm is mixed with viscous blood. Furthermore, the patient is approaching 50 years old. Inborn and postnatal deficit, delayed response time, memory loss, and inexpressiveness are all symptoms of spleen and kidney shortage.

Dizziness is caused by the inability of the liver and kidney Yin to converge Yang, and the hyperactivity of the liver Yang. Liver Yang, phlegm and blood stasis can also be seen in patients with green and black complexion, dark lips and tongue, yellow and greasy coated tongue, sublingual vein circuitous, as well as deep pulse and slippery string. Therefore, the treatment focuses on clearing liver Yang and promoting blood circulation and resolving phlegm, with dried orange peel, Rhizome Pinelliae Preparata, Semen Persicae and Flos Carthami as the monarch drug. Dried orange peel can also regulating Qi of liver and spleen, Qi circulation leads to phlegm elimination and blood circulation leads to phlegm self-transformation. Acorus tatarinowii dry wet phlegm, paeonia suffruticosa cool blood stasis, Rhizoma gastrodia, uncaria rhynchophylla, Radix Puerariae clear the liver and calm the liver to reduce blood pressure. Phlegm turbid evil depressed for a long time and became heat, which can be supported by Bulbus Fritillariae Thunbergii and arisaema cum bile to clear phlegm heat. Prolonged presence of evil in the body depletes healthy Qi, which can be supported by radix stemonae, Rhodiola rosea to tonify deficiency and reduce phlegm. It can also be supported by ground beetles, leeches, earth worm drugs to take its lifting flexible nature, to break the root of blood stasis, Rhizoma Ligustici, Radix Angelicae Sinensis to active blood circulation and tonify blood, charred triplet to tonify spleen and reduce turbidity. Fructus Gardeniae can be used to clear the heat of triple energizers. It can reach the brain orifices with Rhizoma Ligustici and radix angelicae as the guide.

During the second diagnosis, the symptoms of fire and heat decreased slightly compared with the previous diagnosis, and the symptoms of liver Qi stagnation were obvious. Therefore, Fructus Gardeniae was removed and Radix Curcumae added. And it was still amnesia, so acorus tatarinowii was increased to $10 \mathrm{~g}$ to enlighten and awaken the mind. During the three diagnoses, patients had an obvious dry cough, with Cortex Mori to clear lung and moistening dryness. During the fourth diagnosis, blood stasis of tongue and vein was improved, so Semen Persicae and Flos Carthami were reduced to 8g. The patient has been ill for a long time, and the liver and kidney essence and blood loss. Therefore, during the five diagnosis, Polygonatum Kingianum and Fructus Corni were added to nourish the essence and blood of the liver and kidney. Therefore, the combination of various drugs will clear phlegm, remove blood stasis, clear liver heat, and can be cleaned and applied simultaneously.

\section{Summary}

Vascular dementia is common in the elderly. It has the characteristics of occult onset, progressive sampling progress, long course of disease and poor prognosis, which seriously affects daily life. Professor Li believes 
that in the process of dementia, phlegm turbidity and blood stasis interact with each other to blind the clear orifices. Naotaitong Decoction (Naotaitong granule, which has been developed as an in-hospital preparation) is used for treatment, and Salvia Beverage, earth worm, leech and Rhizoma Ligustici are used to activate blood circulation and remove blood stasis and unblock the brain collaterals. Erchen decoction and acorus tatarinowii can remove phlegm and dampness, open orifices and awaken the brain. Polygonatum Kingianum, Fructus Corni and deer-horn glue can be supplemented to replenish the kidney and fill the lean pulp. Herba Lycopi, Leonurus and Radix Achyranthis Bidentatae can be used to reduce blood stasis and induce diuresis. The whole prescription fits the pathogenesis and plays the effects of resolving phlegm and removing blood stasis, tonifying the kidney and filling the marrow, benefiting intelligence and waking up the mind.

A number of clinical and experimental studies on Naotaitong granule have been carried out, and the results show that:

(1) Naotaitong granule can increase the content of acetylcholin esterase in cerebral cortex of VD rats and improve synaptic transmission effect, so as to improve the learning memory of VD rats ${ }^{\text {[2] }}$.

(2) Naotaitong granule can improve the body state of model rats, increase the content of somatostatin (SS) in hippocampus, and play an important role in controlling and delaying the occurrence of vascular dementia $^{[23]}$

(3) Naotaitong granule can improve the learning behavior of model rats, reduce the content of NO in cerebral cortex and reduce the injury after cerebral ischemia ${ }^{[24]}$.

(4) The clinical observation of Naotaitong granule in the treatment of vascular dementia was carried out.

It was discovered that Naotaitong granule may increase the score on the basic intelligence state assessment scale, lower whole blood viscosity in patients at various cutting speeds, and successfully improve patients' cognition, memory, dementia degree, and living capacity ${ }^{[25]}$. Professor Li's theory of VD phlegm and blood stasis combination and simultaneous treatment of phlegm and blood stasis was systematically verified in this study, and it was confirmed that Naotaitong Decoction / Granule prescription based on the method of simultaneous treatment of phlegm and blood stasis plays an important role in controlling and delaying the onset of vascular dementia, with no obvious side effects in clinical application.

\section{Acknowledgments}

Special thanks to Prof. Jun Li, the supervisor of this project.

\section{Funding}

Fund Project: Shaanxi excellent talents training project of traditional Chinese medicine (Shaanxi Traditional Chinese Medicine Letter [2020]112); Project of Shaanxi Administration of traditional Chinese medicine (No. 2021-ZZ-LC016).

\section{Disclosure statement}

The author declares no conflict of interest.

\section{References}

[1] Li O, Zhang J, Xu J, 2021, Study on the Mechanism of Xiangxuan Yishen Prescription on Neuronal apoptosis in Vascular Dementia Model Rats. Shaanxi Journal of Traditional Chinese Medicine, 42(06): $687-691+704$.

[2] Zhou S, Yu Z, Luo X, 2020, Research Advances in Vascular Dementia. Neural Injury and Functional 
Reconstruction, 15(12): 729-731.

[3] Xu X, 2021, Observation on the Curative Effect of Bushen Yizhi Xingnao Decoction on Vascular Dementia and Its Effect on MMSE and HDSADL Score of Vascular Dementia. Chinese Journal of Traditional Medical Science and Technology, 28(04): 614-616.

[4] Shi M, Zhao M, Zhang J, et al. Mesh Meta-analysis of Oral Chinese Patent Medicine in the Adjuvant Treatment of Vascular Dementia[J]. Chinese Herbal Medicine, 2021, 52(13): 3994-4006.

[5] Guan Y, Zhong Y, Mao M, et al., 2021, Discussion on the Treatment of Vascular Dementia from "Blood Stasis and Poison.” Jiangsu Journal of Traditional Chinese Medicine, 53(08): 64-67.

[6] Chan ES, Bautista DT, Zhu Y, et al., 2018, Traditional Chinese Herbal Medicine for Vascular Dementia. Cochrane Database Syst Rev. 12(12): CD010284.

[7] Shi Y, Cao C, Han Z, et al., 2019, An Herbal Medicine Formula, Ameliorates Cognitive Deficits via Modulating Metabolism of Beta Amyloid Protein and Neurotrophic Factors in Hippocampus of A $\beta 1$ 42 Induced Cognitive Deficit Mice. Front Pharmacol. 10: 258.

[8] Mo Q, Hao E, Qin W, et al., 2020, Research Progress on Material Basis and Pharmacological Action of Traditional Chinese Medicine for Promoting Blood Circulation and Removing Stasis. Chinese Journal of Experimental Traditional Medical Formulae, 26(01): 205-216.

[9] Du SQ, Wang X R, Xiao L Y, et al., 2016, Molecular Mechanisms of Vascular Dementia: What can be Learned from Animal Models of Chronic Cerebral Hypoperfusion?. Molecular Neurobiology, 54(5): 3670-3682.

[10] Li J, Shi G, Zhang X, 2013, Construction of the Theoretical System of Brain and Viscera in Traditional Chinese Medicine and Its Clinical Application. Journal of Shaanxi College of Traditional Chinese Medicine, 36(03): 5-8.

[11] Li J, 2002, Treatment of Brain Phlegm and Blood Stasis Syndrome. Journal of Shaanxi College of Traditional Chinese Medicine, (02): 1-3.

[12] Lei Y, Li S, Li J, 2010, Brief Analysis of Professor Li Jun's Discussion on the Treatment of Encephalopathy with Phlegm and Blood Stasis. Modern Traditional Chinese Medicine, 30(03): 6-7.

[13] Lin R, 2020, Clinical Effect of Fuyang Warming Kidney Prescription on Vascular Dementia with Yang Deficiency and Phlegm Stasis. Guangxi University of Chinese Medicine.

[14] Fu X, 2017, Clinical Experimental Study on Syndrome Type of Alzheimer's Disease in Traditional Chinese Medicine and Classification of Abnormal Body fluid Typing in Uygur Medicine. Xinjiang Medical University.

[15] Wang K, 2014, Standards of Diagnosis and Treatment. People's Medical Publishing House, Beijing.

[16] Wei Y, 2009, Traditional Chinese Medicinal Research, China Press of Traditional Chinese Medicine, Beijing.

[17] Han H, Gong H, Zhang Y, et al., 2021, Clinical Study of Shenqi Jiangzhuo Decoction in the Treatment of Vascular Dementia with Phlegm and Turbid Obstruction. Global Traditional Chinese Medicine, 14(05): 0595-0597.

[18] Yang C, 2020, Mechanism of Ditan Decoction in the Treatment of Vascular Dementia Model Rats Based on Inflammatory Response, Oxidative Stress and mirNA-124 /BACE1/A $\beta$ Pathway. Hubei University of Chinese Medicine.

[19] Wang J, Shi A, Zhou H, et al., 2021, Effect of Tongqiaobu Decoction on Cognitive Function of Rats with Vascular Dementia. Hunan Journal of Traditional Chinese Medicine, 37(06): 171-173.

[20] Qi M, Wu H, Tong J, et al., 2021, Study on the Mechanism of Danshensu Improving the Cognitive Function of Vascular Dementia Model Mice through PI3K/Akt Signaling Pathway. Zhejiang Journal of Integrated Traditional Chinese and Western Medicine, 31(04): 314-318.

[21] Yu T, 2010, Orthodox Tradition of Medicine. Shanxi Science and Technology Publishing House, 


\section{Taiyuan.}

[22] Chao Y, 2011, General Treatise on the Cause and Symptoms of Diseases. China Medical Science Press, Beijing.

[23] Du K, Li J, 2004, Effects of Naotaitong Granule on Learning and Memory and AchE Content in Cerebral Cortex of Rats with Vascular Dementia. Study Journal of Traditional Chinese Medicine, (11): $2080-2081+2100$.

[24] Zhou H, Yua P, Li J, 2005, Effects of Naotaitong Granule on Body State and SS Content in Hippocampus of Vascular Dementia Rats. Chinese Journal of Basic Medicine in Traditional Chinese Medicine, (08): 583-584.

[25] Zhou H, Yuan P, Li J, 2007, Clinical Observation on the Treatment of Vascular Dementia with Naotaitong Granule. Liaoning Journal of Traditional Chinese Medicine, (02): 176-177.

\section{Publisher's note}

Bio-Byword Scientific Publishing remains neutral with regard to jurisdictional claims in published maps and institutional affiliations 